\title{
Multistage Interference Cancellation with Diversity Reception for QPSK Asynchronous DS/CDMA System over Multipath Fading Channels
}

\author{
Jianfeng Weng, Guoqiang Xue, Tho Le-Ngoc, and Sofiène Tahar \\ Dept. of Electrical \& Computer Engineering, Concordia University, Canada \\ email: $\{$ jfweng, xue, tho, tahar\}@ece.concordia.ca
}

\begin{abstract}
A multistage interference cancellation (MIC) technique with Rake diversity (MIC-Rake) for QPSK asynchronous direct sequence code division multiple access (DS/CDMA) system over frequency selective multipath Rayleigh fading channels is presented. Unlike the conventional MIC, which tries to subtract the lump sum of the multipleaccess interference (MAI) and the self-interference (SI), the MIC-Rake attempts to cancel the MAI and the partial SI, and to treat the residual SI as useful signal for symbol decision. The Rake combining is employed to collect signal replicas over multiple fading paths. The upper and lower bounds on the bit error probability are derived by using a Gaussian approximation. Furthermore, the effect of the channel estimation error is studied. Analysis and simulation show that the MIC-Rake can provide a performance close to the ideal performance of single-user system, and outperforms the conventional MIC even in the presence of channel estimation error.
\end{abstract}

\section{INTRODUCTION}

It is well-known that the DS/CDMA system may suffer from the multiple-access interference (MAI) and the selfinterference (SI) when operating in multipath fading environment. To jointly suppress the MAI and the SI, multiuser detection techniques have been developed [1]. Among them, multistage interference cancellation (MIC) [2] has become a popular approach due to its simplicity and flexibility.

In this paper, we study the performance of the MIC with Rake combining (MIC-Rake) in QPSK asynchronous CDMA system over frequency-selective multipath Rayleigh fading channels. Here, QPSK is considered instead of BPSK [2] due to its advantages such as its ability to achieve the same bit error probability using half the transmission bandwidth and its randomness property which renders the signal more difficult to be detected using feature detectors $[3,4]$. Furthermore, unlike the conventional MIC (MIC-Conv) [2], the MIC scheme considered here does not try to remove the total SI. Instead, the SI is considered to have two parts: the self intersymbol interference (SII) incurred by the multipath components of the previous symbol, and the self current interference (SCI) corresponding to the current symbol. This MIC scheme treats the SCI as an useful component in symbol decision and only tries to removed the SII [5]. The ideal performance of the MIC-Rake for single-user system is presented, and the upper and lower bounds on the performance in a multi-user system are derived using a Gaussian approximation. The effect of channel estimation error is also studied. Analysis and simulation show that the MIC-Rake can provide a performance close to the ideal case of single-user system and outperforms the conventional MIC even in the presence of channel estimation error.

\section{System DESCRIPTION}

Consider QPSK asynchronous DS/CDMA system with $K$ users over frequency-selective multipath Rayleigh fading channels. The transmitted signal for user $k$ is [4]

$$
s_{k}(t)=\sqrt{\frac{2 \rho_{k}}{N}} b_{k}\left(t-T^{(k)}\right) a_{k}\left(t-T^{(k)}\right) e^{j \theta_{k}}
$$

where $\rho_{k}:=E_{k} / N_{o}$ is the normalized signal power, in which $E_{k}$ is the symbol energy for user $k$ and $N_{o}$ is the noise power spectral density. $T^{(k)}$ is the associated time delay of user $k$ that can be either random or deterministic over $\left[0, T_{b}\right) . \theta_{k}$ is the phase shift associated with user $k$ that can be assumed uniformly distributed over $[0,2 \pi) . b_{k}(t)=$ $\sum_{n=-\infty}^{\infty} e^{-j \phi_{n}^{(k)}} P_{T_{b}}\left(t-n T_{b}\right)$ is the information bearing waveform, where $\phi_{n}^{(k)}$ is the data bearing phase taken from the set $\{2 \pi(m-1) / 4, m=1,2, \ldots, 4\}$ and $P_{T}(t)$ is the rectangle waveform with duration $T . a_{k}(t)$ denotes the signature waveform given by

$$
a_{k}(t)=\sum_{i=-\infty}^{\infty} \frac{1}{\sqrt{2}}\left[a_{\imath}^{(k), I}+j a_{i}^{(k), Q}\right] P_{T_{c}}\left(t-i T_{c}\right)
$$

where $a_{i}^{(k), I}=a_{N+i}^{(k), I}$ and $a_{i}^{(k), Q}=a_{N+i}^{(k), Q}$ are respectively the in-phase and quadrature PN codes assigned to user $k$, which are taken from the set $\{-1,1\}$.

The equivalent lowpass impulse response of the channel for user $k$ can be given by [2]

$$
h_{k}(t)=\sum_{l=1}^{L_{k}} \alpha_{l}^{(k)} \delta\left(t-t_{l}^{(k)}\right)
$$

where $L_{k}$ is the number of paths which can be either random or deterministic. $\alpha_{l}^{(k)}$ and $t_{l}^{(k)}$ are respectively the fading parameter and the time delay in the $l$-th path.

The fading parameters $\alpha_{l}^{(k)}$ are assumed to be the zero mean complex-valued Gaussian random variables. Besides, the channel is considered slowly time-varying and could be reliably estimated using a channel estimator. The channel estimate is given by

$$
\hat{\alpha}_{l}^{(k)}=\alpha_{l}^{(k)}+w_{l}^{(k)}
$$

for all $l$ and $k$, where $w_{l}^{(k)}$ is the estimate noise in the $l$-th path for user $k$, assumed to be statistically independent for 
all $l$ and $k$, and complex-valued Gaussian distributed with zero mean and variance $\sigma_{w_{l k}}^{2}$.

At the first part of the receiver, we assume that there are $\sum_{k=1}^{K} L_{k}$ correlators with the conjugate waveforms $\left\{a_{k}^{*}(t-\right.$ $\left.\left.\tau_{l}^{(k)}\right), l=1,2, \ldots, L_{k}\right\}$ to generate the decision variables for $K$ users, where $\tau_{l}^{(k)}:=T^{(k)}+t_{l}^{(k)}$. For user $u$ in the $q$ th fading path, the received signal will be correlated with $\left\{a_{u}^{*}\left(t-\tau_{q}^{(u)}\right)\right\}$ and integrated from $n T_{b}+\tau_{q}^{(u)}$ to $(n+$ 1) $T_{b}+\tau_{q}^{(u)}$. Then, the output signal will be sampled at the time instant $(n+1) T_{b}+\tau_{q}^{(u)}$ and it yields

$$
\begin{aligned}
V_{n, q}^{(u)}= & \sum_{k=1}^{K} \sqrt{2 \rho_{k}} \sum_{l=1}^{L_{k}} \alpha_{l}^{(k)}\left[e^{-j \phi_{n-m-1}^{(k)}} R_{k, u}\left(\tau_{k, l ; u, q}^{\prime}\right)\right. \\
& \left.+e^{-j \phi_{n-m}^{(k)}} \hat{R}_{k, u}\left(\tau_{k, l ; u, q}^{\prime}\right)\right]+\eta_{n, q}^{(u)}
\end{aligned}
$$

where $m:=\left\lfloor\left(\tau_{l}^{(k)}-\tau_{q}^{(u)}\right) / T_{b}\right\rfloor$, and $\tau_{k, l ; u, q}^{\prime}:=\left(\tau_{l}^{(k)}-\right.$ $\left.\tau_{q}^{(u)}\right)-m T_{b} . R_{k, u}(\tau)=\frac{1}{N} \int_{0}^{\tau} a_{k}(t-\tau) a_{u}^{*}(t) d t$ and $\hat{R}_{k, u}(\tau)=$ $\frac{1}{N} \int_{\tau}^{T_{b}} a_{k}(t-\tau) a_{u}^{*}(t) d t$ are the normalized partial correlation functions. $\eta_{n, q}^{(u)}$ is the noise component given by

$$
\eta_{n, q}^{(u)}=\frac{1}{\sqrt{N}} \int_{n T_{b}+\tau_{q}^{(u)}}^{(n+1) T_{b}+\tau_{q}^{(u)}} z(t) a_{u}^{*}\left(t-\tau_{q}^{(u)}\right) d t
$$

where $z(t)$ is the background Gaussian noise with zero mean and unit power spectral density. Hence, $\eta_{n, q}^{(u)}$ is Gaussian with zero mean and unit variance. The covariance of $\eta_{n_{1}, q_{1}}^{\left(u_{1}\right)}$ and $\eta_{n_{2}, q_{2}}^{\left(u_{2}\right)}$ can be given by

$$
\begin{aligned}
& \frac{1}{2} E\left\{\eta_{n_{1}, q_{1}}^{\left(u_{1}\right)}\left[\eta_{n_{2}, q_{2}}^{\left(u_{2}\right)}\right]^{*}\right\}= \\
& \begin{cases}0 & \text { if } \tau_{2}^{S}>\tau_{1}^{L} \\
0 & \text { if } \tau_{1}^{S}>\tau_{2}^{L} \\
R_{u_{2}, u_{1}}\left(\tau_{2}^{L}-\tau_{1}^{S}\right) & \text { if } \tau_{1}^{S} \leq \tau_{2}^{L} \leq \tau_{1}^{L} \\
R_{u_{1}, u_{2}}^{*}\left(\tau_{1}^{L}-\tau_{2}^{S}\right) & \text { if } \tau_{2}^{S} \leq \tau_{1}^{L} \leq \tau_{2}^{L}\end{cases}
\end{aligned}
$$

where $\tau_{i}^{S}=n_{i} T_{b}+\tau_{q_{2}}^{\left(u_{2}\right)}$ and $\tau_{\imath}^{L}=\tau_{i}^{S}+T_{b}$ for $i=1,2$.

The conventional Rake combiner [3] can be used to collect the fading replicas $\left\{V_{n, q}^{(u)}\right\}$. During the detection, all other multiuser and multipath signals are treated as interference. Therefore, although this approach is quite simple, the performance of the Rake combiner would degrade due to the detrimental effect of the interferences from multiple users and multiple paths. To alleviate the detriment, a multistage interference cancellation (MIC) [2] scheme can be applied.

\section{MIC WITH DIVERSITY}

Conventional MIC with diversity: The MIC scheme over multipath fading has been addressed in [2]. To facilitate our discussion, we may rewrite $V_{n, q}^{(u)}$ of (5) into

$V_{n, q}^{(u)}=\sqrt{2 \rho_{u}} \alpha_{q}^{(u)} e^{-j \phi_{n}^{(u)}}+S c_{n, q}^{(u)}+S i_{n, q}^{(u)}+M_{n, q}^{(u)}+\eta_{n, q}^{(u)}$ where $S c_{n, q}^{(u)}, S i_{n, q}^{(u)}$, and $M_{n, q}^{(u)}$ are respectively defined as

$$
\begin{gathered}
S c_{n, q}^{(u)}=\sqrt{2 \rho_{u}} \sum_{l=1, l \neq q}^{L_{u}} \alpha_{l}^{(u)} e^{-j \phi_{n}^{(u)}} \ddot{R}_{u, u}\left(\tau_{u, l ; u, q}^{\prime}\right) \\
S i_{n, q}^{(u)}=\sqrt{2 \rho_{u}} \sum_{l=1, l \neq q}^{L_{u}} \alpha_{l}^{(u)}\left[d_{m+1} e^{-j \phi_{n-m-1}^{(u)}}\right. \\
\left.R_{u, u}\left(\tau_{u, l ; u, q}^{\prime}\right)+d_{m} e^{-j \phi_{n-m}^{(u)}} \hat{R}_{u, u}\left(\tau_{u, l ; u, q}^{\prime}\right)\right] \\
M_{n, q}^{(u)}=\sum_{k=1, k \neq u}^{K} \sqrt{2 \rho_{k}} \sum_{l=1}^{L_{k}} \alpha_{l}^{(k)}\left[e^{-j \phi_{n-m-1}^{(k)}}\right. \\
\left.R_{k, u}\left(\tau_{k, l ; u, q}^{\prime}\right)+e^{-j \phi_{n-m}^{(k)}} \hat{R}_{k, u}\left(\tau_{k, l, u, q}^{\prime}\right)\right]
\end{gathered}
$$

In (10), $\ddot{R}_{u, u}(\tau)$ is set to either $R_{u, u}(\tau)$ or $\hat{R}_{u, u}(\tau)$ depending on $\tau_{l}^{(u)}<\tau_{q}^{(u)}$ or not. $d_{i}$ is an index function which can be set to 0 if $i=0$ and to 1 otherwise. $S c_{n, q}^{(u)}$ is referred to as the self current-symbol interference (SCI) and $S i_{n, q}^{(u)}$ the self intersymbol interference (SII). The sum of the SCI and the SII is the total SI.

As shown in [2], in each stage of the MIC, the MAI and the SI (both the SII and the SCI) can be reconstructed and subtracted from the received signal before the symbol decision. For such an MIC, the output of the canceller in the $i$-th stage can be given by

$$
\begin{aligned}
V_{n, q}^{(u)}(i)= & V_{n, q}^{(u)}-\hat{S} c_{n, q}^{(u)}(i-1)-\hat{S} i_{n, q}^{(u)}(i-1) \\
& -\hat{M}_{n, q}^{(u)}(i-1)
\end{aligned}
$$

where $\hat{S} c_{n, q}^{(u)}(i-1), \hat{S} i_{n, q}^{(u)}(i-1)$, and $\hat{M}_{n, q}^{(u)}(i-1)$ are the reconstructed interference parts in the $(i-1)$-th stage and they can be respectively expressed by (9), (10), and (11) with $\phi_{n}^{(k)}$ and $\alpha_{l}^{(k)}$ being replaced by the tentative decision $\hat{\phi}_{n}^{(k)}(i-1)$ and the channel estimate $\hat{\alpha}_{l}^{(k)}$, respectively.

After removing the MAI and the SI, the Rake combining can be applied to collecting the signal replicas. The test statistic is given by

$$
X_{n}^{(u)}(i)=\left[\underline{\hat{\alpha}}^{(u)}\right]^{H} \underline{V}_{n}^{(u)}(i)
$$

where $\underline{V}_{n}^{(u)}(i)=\left[V_{n, 1}^{(u)}(i), V_{n, 2}^{(u)}(i), \ldots, V_{n, L_{u}}^{(u)}(i)\right]^{T}, \underline{\hat{\alpha}}^{(u)}=$ $\left[\hat{\alpha}_{1}^{(u)}, \hat{\alpha}_{2}^{(u)}, \ldots, \hat{\alpha}_{L_{u}}^{(u)}\right]^{T}$.

Finally, we compute the following decision variables for QPSK $(M=4)$,

$$
D_{p}(i)=\operatorname{Re}\left\{X_{n}^{(u)}(i) e^{\jmath \psi_{p}}\right\}, \quad p=1,2, \ldots, M
$$

where $\psi_{p}:=2 \pi(p-1) / M$, and then make symbol decision according to

$$
\hat{\phi}_{n}^{(u)}(i)=\psi_{p}=\operatorname{argmax}\left\{D_{p}(i)\right\}
$$


For simplicity, in the case of $i=0$, we let $V_{n, q}^{(u)}(0)=V_{n, q}^{(u)}$. As such, the tentative decisions made in the initial stage are equivalent to those in the conventional Rake combiner.

In the above conventional MIC (MIC-Conv), the SCI is regarded as an interference to be subtracted in (12). Nevertheless, the SCI actually contains the current symbol, which is useful for symbol decision. As an illustration, considering the received signal in the first arriving path $V_{n, 1}^{(u)}(i)$ and supposing that all the MAI and the SII parts have been subtracted from the received signal, we have

$$
\begin{aligned}
V_{n, 1}^{(u)}(i)= & \sqrt{2 \rho_{u}} \alpha_{1}^{(u)} e^{-j \phi_{n}^{(u)}}+\sqrt{2 \rho_{u}} \sum_{l=2}^{L_{u}} \alpha_{l}^{(u)} \\
& e^{-j \phi_{n}^{(u)}} \ddot{R}_{u, u}\left(\tau_{u, l ; u, 1}^{\prime}\right)+\eta_{n, 1}^{(u)}
\end{aligned}
$$

If we disregard the received signals from other paths and assume the perfect channel knowledge, the test statistic $X$ for the optimum demodulation is not given by $\left[\alpha_{1}^{(u)}\right]^{*} V_{n, 1}^{(u)}(i)$. Rather, it would be $X=\left[\beta_{1}^{(u)}\right]^{*} V_{n, 1}^{(u)}(i)$, i.e.,

$$
X=\left[\beta_{1}^{(u)}\right]^{*}\left[\beta_{1}^{(u)} e^{-j \phi_{n}^{(u)}}+\eta_{n, 1}^{(u)}\right]
$$

where $\beta_{1}^{(u)}=\sqrt{2 \rho_{u}} \alpha_{1}^{(u)}+\sqrt{2 \rho_{u}} \sum_{l=2}^{L_{u}} \alpha_{l}^{(u)} \ddot{R}_{u, u}\left(\tau_{u, l ; u, 1}^{\prime}\right)$.

By using (17), the SCI part in (16) is treated as a useful signal for symbol decision rather than the interference in the SCI subtraction. In this sense, the resulting performance would be better.

New MIC with Rake diversity: In the new MIC with Rake (MIC-Rake), we propose not to subtract the SCI part because it contains the useful signal. Thus, the output of the canceller in the $i$-th stage for user $u$ is given by

$$
V_{n, q}^{(u)}(i)=V_{n, q}^{(u)}-\hat{S} i_{n, q}^{(u)}(i-1)-\hat{M}_{n, q}^{(u)}(i-1)
$$

where $\hat{S} i_{n, q}^{(u)}(i-1)$ and $\hat{M}_{n, q}^{(u)}(i-1)$ are the reconstruction of the SII and the MAI as in the conventional MIC.

Relying on $V_{n, q}^{(u)}(i)$, the tentative symbol decision for $\phi_{n}^{(u)}$ can be followed by (13)-(15).

\section{PERFORMANCE ANALYSIS}

\section{A. Ideal performance in single-user case}

Assume equiprobable transmitted symbols and Gray code bit mapping. By ignoring the SII and the MAI, the canceller output can be written as

$$
\underline{V}_{n}^{(u)}(i)=\mathbf{R}_{s} \underline{\alpha}^{(u)} \sqrt{2 \rho_{u}} e^{-j \phi_{n}^{(u)}}+\underline{\eta}_{n}^{(u)}
$$

where $\underline{\eta}_{n}^{(u)}=\left[\eta_{n, 1}^{(u)}, \eta_{n, 2}^{(u)}, \ldots, \eta_{n, L_{u}}^{(u)}\right]^{T} . \mathbf{R}_{s}$ is an $L_{u} \times L_{u}$ correlation matrix of the signature waveforms. Its $i j$-th entry is set to $\hat{R}_{u, u}\left(\tau_{j}^{(u)}-\tau_{\imath}^{(u)}\right)$ if $\tau_{\jmath}^{(u)} \geq \tau_{i}^{(u)}$ and $R_{u, u}\left(T_{b}+\right.$ $\left.\tau_{j}^{(u)}-\tau_{i}^{(u)}\right)$ otherwise. By (7), the correlation matrix of $\underline{\eta}_{n}^{(u)}$ can be recognized to be $\mathbf{R}_{s}$.

Let $\mathbf{R}_{s}=\mathbf{Q}_{s} \Lambda_{s} \mathbf{Q}_{s}^{H}$, where $\mathbf{Q}_{s}$ is the orthogonal matrix and $\Lambda_{s}=\operatorname{diag}\left(\lambda_{1}, \lambda_{2}, \ldots, \lambda_{L_{u}}\right)$ is the diagonal matrix, in which $\left\{\lambda_{q}\right\}$ are the eigenvalues of $\mathbf{R}_{s}$. Thus, the test statistic following (13) can be written as

$$
X=\left[\underline{\hat{\beta}}^{(u)}\right]^{H}\left[\underline{\beta}^{(u)} \sqrt{2 \rho_{u}} e^{-j \phi_{n}^{(u)}}+\underline{\xi}_{n}^{(u)}\right]
$$

where $\underline{\beta}^{(u)}:=\Lambda_{s}^{\frac{1}{2}} \mathbf{Q}_{s}^{H} \underline{\alpha}^{(u)}, \underline{\hat{\beta}}^{(u)}:=\Lambda_{s}^{\frac{1}{2}} \mathbf{Q}_{s}^{H} \underline{\hat{\alpha}}^{(u)}$ and $\underline{\xi}^{(u)}:=\Lambda_{s}^{-\frac{1}{2}}$ $\mathrm{Q}_{s}^{H} \underline{\eta}_{n}^{(u)}$. Here, $n,(u)$, and $(i)$ of $X_{n}^{(u)}(i)$ are omitted.

Thus, the bit error rate(BER) can be given by [6]

$$
P_{b}=\mathcal{F}(\gamma=-\pi / 4)=-\sum_{\left\{\omega_{p}\right\}} \operatorname{Res}\left[\Phi_{\gamma}(\omega) / \omega, \omega_{p}\right]
$$

where $\mathcal{F}(\gamma):=\operatorname{Pr}\left\{\operatorname{Re}\left\{X e^{j \gamma}\right\}<0\right\}$ denotes the probability that $\operatorname{Re}\left\{X e^{j \gamma}\right\}$ is less than $0 . \Phi_{\gamma}(\omega)$ denotes the characteristic function (CF) of $\operatorname{Re}\left\{X e^{j \gamma}\right\} .\left\{\omega_{p}\right\}$ is the set of poles of $\Phi_{\gamma}(\omega) / \omega$ in the upper half plane $(\operatorname{Im}(\omega)>0)$ and $\operatorname{Res}\left[f(\omega), \omega_{p}\right]$ is the residue of $f(\omega)$ at $\omega=\omega_{p}$.

In evaluating $\Phi_{\gamma}(\omega)$, we let $\mathbf{R}_{\beta}=\frac{1}{2} E\left\{\beta^{(u)}\left[\beta^{(u)}\right]^{H}\right\}$ and similarly for $\mathbf{R}_{s}$, we diagonalize $\mathbf{R}_{\beta}$ so that $\mathbf{R}_{\beta}=\mathbf{Q}_{\beta} \Lambda_{\beta} \mathbf{Q}_{\beta}^{H}$. Then, substituting (4) into (20), we have

$$
X=C^{H} C \sqrt{2 \rho_{u}}+C^{H}\left(G \sqrt{2 \rho_{u}}+Z\right)+G^{H} Z
$$

where $C:=\mathbf{Q}_{\beta}^{H} \underline{\beta}^{(u)}, G:=\mathbf{Q}_{\beta}^{H} \Lambda_{s}^{\frac{1}{2}} \mathbf{Q}_{s}^{H} \Delta \underline{\alpha}^{(u)}$ and $Z:=\mathbf{Q}_{\beta}^{H} \underline{\xi}_{n}^{(u)}$. $\Delta \underline{\alpha}^{(u)}=\underline{\hat{\alpha}}^{(u)}-\underline{\alpha}^{(u)}$.

We note that the elements in the vector $C$ are statistically independent, so are those in the vector $Z$. However, the elements in $G$ may be correlated.

In evaluating the CF of $\operatorname{Re}\left\{X e^{j \gamma}\right\}$, we first assume $\frac{1}{2} E\{$ $\left.G G^{H}\right\}=\lambda^{(G)}$ I. The CF of $\operatorname{Re}\left\{X e^{j \gamma}\right\}$ can be given by

$$
\Phi_{\gamma}(\omega)=\prod_{q=1}^{L_{u}} \frac{1}{a_{q}^{2} \omega^{2}-2 j \omega b_{q}+1}
$$

Here, $a_{q}:=\sqrt{\left[\lambda^{(Z)}+2 \rho_{u} \lambda^{(G)}\right] \sigma_{q}^{2}+\lambda^{(Z)} \lambda^{(G)}}$ and $b_{q}:=$ $\sqrt{2 \rho_{u}} \cos \gamma \sigma_{q}^{2}$.

Using $\Phi_{\gamma}(\omega)$, the BER can be calculated via (21). Generally, the explicit expression of $\mathcal{F}(\gamma)$ depends on the order of the poles and is very complicated. In our case, the eigenvalues are usually distinct and thus we have

$$
P_{b}\left(\lambda^{(G)}, \lambda^{(Z)}\right)=\sum_{q=1}^{L_{u}} \pi_{q} \frac{1}{2}\left[1-\sqrt{\frac{b_{q}^{2} / a_{q}^{2}}{1+b_{q}^{2} / a_{q}^{2}}}\right]
$$

where

$$
\pi_{q}=\left.\prod_{l=1, l \neq q}^{L_{u}} \frac{1}{a_{l}^{2} \omega^{2}-2 j \omega b_{l}+1}\right|_{\omega=j\left(b_{q} / a_{q}+\sqrt{1+b_{q}^{2} / a_{q}^{2}}\right) / a_{q}}
$$


Here $a_{q}:=\sqrt{\left[\lambda^{(Z)}+2 \rho_{u} \lambda^{(G)}\right] \sigma_{q}^{2}+\lambda^{(Z)} \lambda^{(G)}}$ and $b_{q}:=\sqrt{\rho_{u}}$ $\sigma_{q}^{2}$.

For correlated $G$, to evaluate the CF of $\operatorname{Re}\left\{X e^{j \gamma}\right\}$ is quite difficult due to the noise terms $C^{H} G$ and $G^{H} Z$ in (22). Note that at SNR values of practical interest, the term $G^{H} Z$ is relatively small in comparison with the noise term $C^{H}\left(G \sqrt{2 \rho_{u}}+Z\right)$ [3]. Hence, the upper and lower bounds on the bit error probability can be derived and they are corresponding to the maximum and minimum variances of the noise $C^{H} G \sqrt{2 \rho_{u}}$, respectively. Furthermore, note that conditioning on $C$ we have $\lambda_{\min }^{\left(G^{\prime}\right)} C^{H} C \leq C^{H} \frac{1}{2} E\left\{G G^{H}\right\} C \leq$ $\lambda_{m a x}^{(G)} C^{H} C$, where $\lambda_{m a x}^{(G)}$ and $\lambda_{m i n}^{(G)}$ are the maximum and minimum eigenvalues of the covariance matrix $\frac{1}{2} E\left\{G G^{H}\right\}$, respectively. Therefore, the upper and lower bounds for the BER can be obtained by setting $\frac{1}{2} E\left\{G G^{H}\right\}$ to $\lambda_{\max }^{(G)} \mathbf{I}$ and $\lambda_{\min }^{(G)} \mathrm{I}$, respectively. Specifically, we have

$$
\begin{aligned}
& P_{b}^{(\text {up })}=P_{b}\left(\lambda_{\max }^{(G)}, 1\right) \\
& P_{b}^{(\text {low })}=P_{b}\left(\lambda_{\min }^{(G)}, 1\right)
\end{aligned}
$$

where $P_{b}\left(\lambda^{(G)}, \lambda^{(Z)}\right)$ is the function in (24) and $\lambda^{(Z)}$ is now set to 1 because the noise vector has already been normalized in (20) and (22).

The above BER expression is valid for both perfect and imperfect channel knowledge. In the case of perfect channel knowledge, one can readily check that by setting $\lambda^{(G)}=0$ and $\lambda^{(Z)}=1, P_{b}^{(u p)}$ and $P_{b}^{(l o w)}$ are the same and (24) simply reduces to eq.14-5-28 of [3].

\section{B. Performance in multi-user system}

In the $i$-th stage, we assume that the residual interference terms, $\tilde{S} i_{n, q}^{(u)}(i-1):=S i_{n, q}^{(u)}-\hat{S i}_{n, q}^{(u)}(i-1)$ and $\tilde{M}_{n, q}^{(u)}(i-$ $1):=M_{n, q}^{(u)}(i)-\hat{M}_{n, q}^{(u)}(i-1)$, are approximately Gaussian distributed for large $K$ and $L_{k}$ [2]. The test statistic $X(i)$ can be written as

$$
X(i)=[C+G]^{H}\left[C \sqrt{2 \rho_{u}}+F(i)+Z\right]
$$

where $F(i):=\mathbf{Q}_{\beta}^{H} \Lambda_{s}^{-\frac{1}{2}} \mathbf{Q}_{s}^{H}\left(\underline{\tilde{S}}_{n}^{(u)}(i-1)+\underline{\tilde{M}}_{n}^{(u)}(i-1)\right)$ related to the residual interference. $\underline{\tilde{S}}_{n}^{(u)}(i-1)$ and $\underline{\tilde{M}}_{n}^{(u)}(i-$ 1) are respectively the vectors of $\tilde{S} i_{n, q}^{(u)}(i-1)$ and $\tilde{M}_{n, q}^{(u)}(i-$ 1) for $q=1,2, . ., L_{u}$.

Consider user $u$. Let $\mathbf{R}_{F}(i)$ denote the covariance matrix of $F(i)$. For $i>0$, the evaluation of $\mathbf{R}_{F}(i)$ is dependent on the BER in the previous stage $(i-1)$, of which the exact result is unfortunately difficult to be obtained and the upper and lower bounds are derived. Therefore, we further use $\mathbf{R}_{F}^{(u p)}(i)$ to denote the covariance matrix of $F(i)$ evaluated from $P_{b}^{(u p)}(i-1)$ and $\mathbf{R}_{F}^{(l o w)}(i)$ as the one evaluated from $P_{b}^{(l o w)}(i-1)$. In the initial stage $i=0$, however, we may set $\hat{S}_{n, q}^{(u)}(i-1)$ and $\hat{M}_{n, q}^{(u)}(i-1)$ to zero

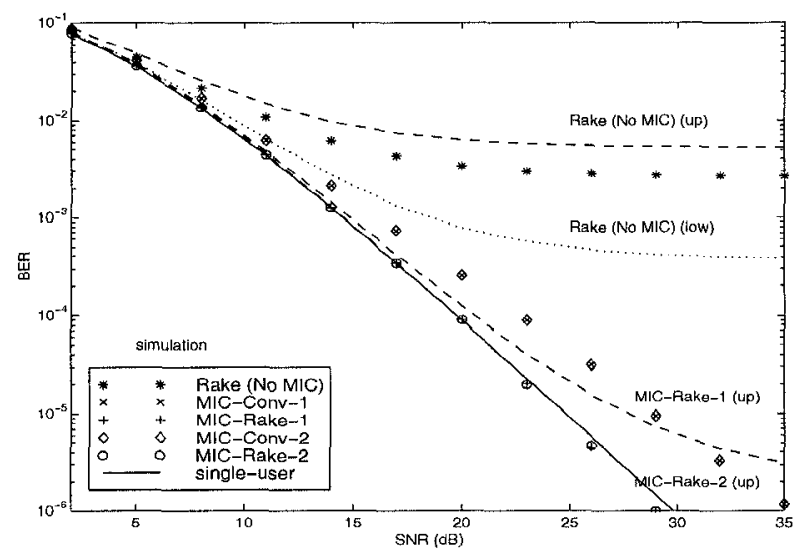

Fig. 1. Analytical and simulation results on the BER of the MIC versus SNR in a 3-user system with $N=31$ over 2-path Rayleigh fading channels. Equal-power users. Dashed lines: upper bound, dotted line: lower bound.

and directly evaluate $\mathbf{R}_{F}(0)$. Here, for consistency, we let $\mathbf{R}_{F}^{(u p)}(0)=\mathbf{R}_{F}^{(l o w)}(0)=\mathbf{R}_{F}(0)$.

Let $\lambda_{m a x}^{(F, u p)}(i)$ denote the maximum eigenvalue of $\mathbf{R}_{F}^{(u p)}(i)$ and $\lambda_{\min }^{(F, \text { low })}(i)$ the minimum eigenvalue of $\mathbf{R}_{F}^{(l o w)}(i)$. Similarly as in the single-user case, the upper and lower bounds of the BER in the $i$-th stage are respectively given by

$$
\begin{aligned}
& P_{b}^{(u p)}(i)=P_{b}\left(\lambda_{\max }^{(G)}, 1+\lambda_{\max }^{(F, u p)}(i)\right) \\
& P_{b}^{(l o w)}(i)=P_{b}\left(\lambda_{\min }^{(G)}, 1+\lambda_{\min }^{(F, l o w)}(i)\right)
\end{aligned}
$$

\section{NUMERICAL AND SIMULATION RESULTS}

System model: Identical fading channels with $E\left\{\left[\alpha_{l}^{(k)}\right]^{2}\right\}=$ 2 for all fading paths are considered, for which $\mathbf{R}_{\alpha}=\mathbf{I}$. It is noteworthy to mention that our analysis is valid for arbitrary $\mathbf{R}_{\alpha}$ and applicable to unbalanced fading channels. For user $k$, the total SNR\# $k$ per bit over the $L_{k}$ fading channels is set to $\frac{1}{2} L_{k} 2 \rho_{k} \mathrm{E}\left\{\left[\alpha_{l}^{(k)}\right]^{2}\right\} / \log _{2} M=\rho_{k} L_{k} . L_{k}$ is assumed same for all users and denoted by $L$. Gold sequences with $N=31$ are considered as the PN codes. For any user $k$, we let $t_{l}^{(k)}=l T_{c} / 2$ and $T^{(k)}=(k-1) T_{b} / 10 \bmod T_{b}$, where without loss of generality $T^{(k)}$ is arranged within $\left[0, T_{b}\right)$. The simulated BER is estimated from $3 \times 10^{6}$ Monte Carlo trials.

Results: The analytical and simulation results on the BER for the MIC in a 3-user system over 2-path Rayleigh fading channels is plotted versus SNR in Fig. 1 for $N=31$. All users are considered with equal power and perfect channel knowledge is assumed. In the figure, the single-user performance is evaluated via (26) with $\lambda_{\max }^{(G)}=0$ due to the perfect channel knowledge. The upper bounds for the Rake (No MIC) and the MIC-Rake-1,2 are evaluated via (29). The lower bound for the Rake is evaluated via the (30). The other BERs are obtained from the simulation. It can be seen that both the MIC-Rake-1,2 and the MIC-Conv-1,2 


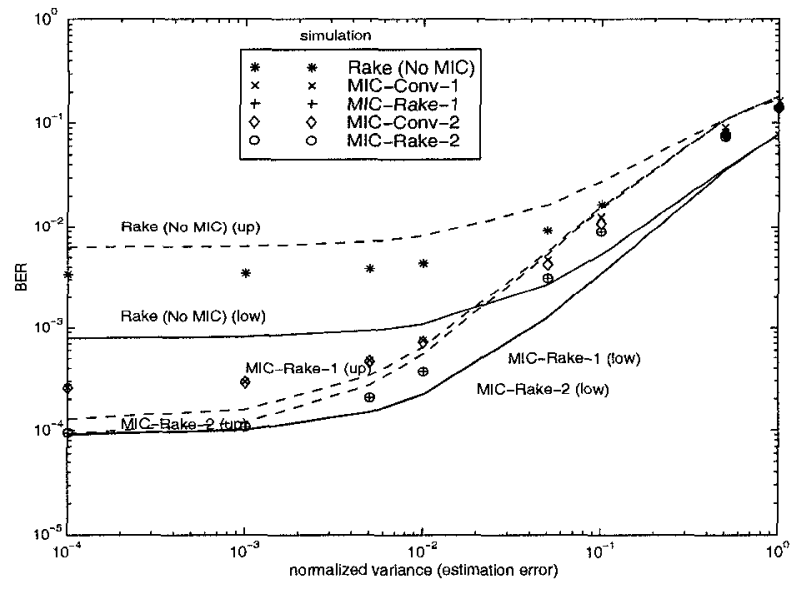

Fig. 2. Analytical and simulation results on the BER of the MIC versus the variance of the channel estimation error in a 3-user system over 2 path Rayleigh fading channels. SNR=20dB, $N=31$, and equal-power users. Dashed lines: upper bound, solid lines: lower bound.

can provide significant performance improvement over the conventional Rake receiver. Furthermore, by increasing the number of stages from 1 to 2 , the performance improvement is negligible while by using the MIC-Rake instead of the MIC-Conv, the BER can be further decreased and a near single-user performance achieved. In particular, at the BER level of $10^{-3}$, approximately $2 \mathrm{~dB}$ SNR improvement can be achieved by the MIC-Rake over the MIC-Conv. Furthermore, from Fig. 1, the simulation results are shown well upper bounded by the corresponding analytical results.

Next, we consider the case of the channel estimation error. We assume that the variance of the channel estimation error in (4) is the same for all $l$ and $k$ and can be denoted by $\sigma_{w}^{2}$. In Fig.2, we compare the analytical and simulation results on the bit error rates of the MIC versus $\sigma_{w}^{2}$ in 3-user system with $\mathrm{SNR}=20 \mathrm{~dB}$ and $N=31$ over 2-path Rayleigh fading channels. All users are considered with equal power. The upper and lower bounds for the Rake and the MICRake-1,2 are obtained via (29) and (30), respectively. Other BERs are estimated via simulation. It can be seen that when the variance of the channel estimation noise is less than $10^{-2}$, both the MIC-Rake-1,2 and the MIC-Conv-1,2 can still provide a significant performance improvement over the conventional Rake. As the variance of the estimation noise increases, the performance difference would become small. Nevertheless, it is shown in Fig. 2 that even in the presence of the estimation noise the MIC-Rake still outperforms the conventional one (MIC-Conv).

Further simulation results and the corresponding upper and lower bounds on the bit error probability of the MIC versus the number of users $(K)$ at $\mathrm{SNR}=20 \mathrm{~dB}$ over 2-path Rayleigh fading channels are plotted in Fig. 3, where $\sigma_{w}^{2}=10^{-2}$ and all users are with equal power. Once again, it can be seen that the simulation results are well upper and lower bounded by the analytical ones. Besides, it can be seen that

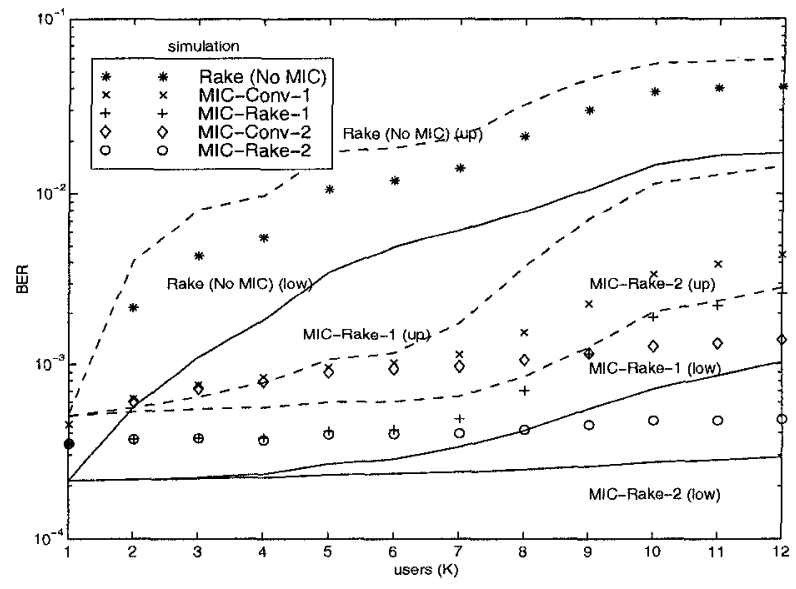

Fig. 3. Analytical and simulation results on the BER of the MIC versus the number of users $(K)$ in multi-user system over 2-path Rayleigh fading channels. SNR $=20 \mathrm{~dB}, N=31$, and equal-power users. $\sigma_{w}^{2}=10^{-2}$. Dashed lines: upper bound, solid lines: lower bound.

as $K$ increases, the performances of both the MIC-Rake-1 and the MIC-Conv-1 are degraded. Also, a slight performance degradation occurs for the 2-stage MIC (MIC-Rake2 and MIC-Conv-2). This phenomenon is due to the disturbance of the channel estimation error. The more users, the more residual interference even if all the tentative decisions in the previous stage are correct. Furthermore, the results show that in the case of imperfect channel knowledge, the MIC-Rake still performs better than the MIC-Conv. In particular, if we set BER $=10^{-3}$ as an acceptable performance level, by using the MIC-Conv-1 and 2, the system can only accommodate 7 and 9 active users, respectively. In contrast, by using the MIC-Rake, the system can tolerate 9 active users in the MIC-Rake-1 and at least 12 users in the MIC-Rake-2.

\section{ACKNOWLEDGMENT}

This work is partially supported by Ericsson Research Canada.

\section{REFERENCES}

[1] S. Verdu, "Minimum probability of error for asynchronous Gaussian multiple access channels," IEEE Trans. Inform. Theo., vol. IT-32, pp. 85-96, Jan. 1986.

[2] Y. C. Yoon, R. Kohno, and H. Imai, "Cascaded co-channel interference cancelling and diversity combining for spread spectrum multiaccess over multipath fading channels," IEICE Trans. Commun, vol. E76-B, pp. 163-168, Feb. 1993.

[3] J. G. Proakis, Digital communications. New York: McGraw-Hill, 1995.

[4] R. L. Peterson, R. E. Ziemer, and D. E. Borth, Introduction to spread spectrum communications. London: Prentice-Hall, Inc., 1995.

[5] J. F. Weng, G. Q. Xue, T. Le-Ngoc, and S. Tahar, "Multistage interference cancellation with multipath decorrelating for QPSK asynchronous DS/CDMA system over multipath fading," in VTC99, Houston, Texas, May 16-22 1999.

[6] P. Y. Kam, "Bit error probability of MDPSK over the non-selective Rayleigh fading channels with diversity reception," IEEE Trans. Commun., vol. COM-39, pp. 220-224, Feb. 1991. 